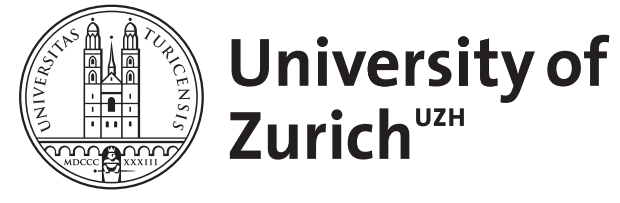

\title{
Der Gesellschaftsvertrag als Chimäre und rationale Utopie
}

\author{
Marti-Brander, Urs
}

DOI: https://doi.org/10.5771/9783845293325-145

Posted at the Zurich Open Repository and Archive, University of Zurich

ZORA URL: https://doi.org/10.5167/uzh-162294

Book Section

Accepted Version

Originally published at:

Marti-Brander, Urs (2018). Der Gesellschaftsvertrag als Chimäre und rationale Utopie. In: Lau, Thomas; Reinhardt, Volker; Voigt, Rüdiger. Der Bürger als Souverän : Jean-Jacques Rousseaus Lehre von der volonté générale [im Spiegel der Zeit]. Baden-Baden: Nomos, 145-166.

DOI: https://doi.org/10.5771/9783845293325-145 
Erscheint in: Rüdiger Voigt, Volker Reinhardt, Thomas Lau (Hg.), Der Bürger als Souverän. Jean-Jacques Rousseaus Lehre von der volonté générale, Baden-Baden, 2018.

\section{Urs Marti-Brander}

\section{Der Gesellschaftsvertrag als Chimäre und rationale Utopie}

J'ouvre les livres de droit et de morale, j'écoute les savans et les jurisconsultes et pénétré de leurs discours insinuans, je déplore les misères de la nature, j'admire la paix et la justice établies par l'ordre civil, je bénis la sagesse des institutions publiques et me console d'être homme en me voyant citoyen. Bien instruit de mes devoirs et de mon bonheur, je ferme le livre, sors de la classe, et regarde autour de moi; je vois des peuples infortunés gemissans sous un joug de fer, le genre humain ecrasé par une poignée d'oppresseurs, une foule affamée, accablée de peine et de faim, dont le riche boit en paix le sang et les larmes, et partout le fort armé contre le foible du redoutable pouvoir des loix.

Rousseau, OC III, 608-609. ${ }^{1}$

Ich möchte in meinem Beitrag in drei Schritten darlegen, wie Rousseau selbst seiner theoretischen Konstruktion des Contrat social systematisch immer wieder den Boden entzieht. Im ersten Schritt geht es um die Schwierigkeit, das Individuum vom Nutzen der Gemeinschaft und der Notwendigkeit, sich deren Regeln zu unterwerfen, zu überzeugen. Danach wird erörtert, wie die Spaltung der Gesellschaft zwischen Reich und Arm, Macht und Ohnmacht die Menschen korrumpiert und die Republik unterminiert. Schließlich ist daran zu erinnern, dass Rousseau erklärt hat, die Zeit der Republiken gehöre in Europa der Vergangenheit an.

\section{Sich von sich selbst separieren}

Wie plausibel ist die Annahme, politische Gemeinschaften beruhten auf einem Gesellschaftsvertrag? Die Frage bedarf der Präzisierung: Einerseits interessiert, ob in der Geschichte nachweislich Verträge abgeschlossen worden sind, auf die Staaten sich berufen können, um ihre Legitimität zu begründen. Andererseits gilt es zu prüfen, ob angesichts der natürlichen Neigungen der Menschen die Annahme,

\footnotetext{
${ }^{1}$ Rousseau OC III, 608-609; Bloch 2010, 307: „Ich öffne die Bücher über Recht und Moral, ich höre mir die Gelehrten und Rechtskundigen an, und von ihren vereinnahmenden Reden durchdrungen, beklage ich das Elend der Natur, ich bewundere den Frieden und die Gerechtigkeit, die durch die zivile Ordnung errichtet werden, ich preise die Weisheit der öffentlichen Institutionen, und mich als Staatsbürger zu wissen, tröstet mich darüber hinweg, Mensch zu sein. Wohlunterrichtet über meine Pflichten und mein Glück schließe ich das Buch, trete aus der Klasse und schaue um mich: Ich sehe unglückliche Völker unter eisernem Joch ächzen, die gesamte Menschheit von einer Handvoll Unterdrücker gebeugt, eine von Leid gepeinigte und nach Brot hungernde Menge, deren Blut und Tränen der Reiche ungestört trinkt, und überall ist der Starke wider die Schwachen mit der furchtbaren Macht der Gesetze bewaffnet.“
} 
sie könnten sich zusammenfinden, einen Vertrag zwecks gegenseitiger Respektierung von Recht und Freiheit schließen und ihr ganzes Handeln danach richten, aufrecht zu erhalten ist. Hören wir zunächst einige Antworten von verschiedener Seite.

Locke - der weise Locke, wie Rousseau ihn nennt - hält es für eine offenkundige Tatsache, dass die Anfänge von Rom und Venedig sich einem solchen Vertrag verdanken. Er spricht allerdings von einer „Vereinigung verschiedener freier und voneinander unabhängiger Menschen [...] unter denen es keine natürliche Überlegenheit und Unterwerfung gab.“22 Damit hat er, ohne explizit darauf einzugehen, bereits zu verstehen gegeben, dass Menschen, die von anderen abhängig sind - Besitzlose - nicht Vertragspartner sein können. Hume hat Locke widersprochen, für ihn ist nicht einsichtig, weshalb die Legitimität der sozialen Ordnung abhängig sein soll von bewusster Zustimmung oder gemeinwohlorientierter Gesinnung. Zwar räumt er ein, politische Institutionen seien in frühen Zeiten dank Übereinkunft zwischen Menschen zustande gekommen, die sich deren Autorität freiwillig unterworfen hätten. Was aber seine eigene Zeit betrifft, so hält er eine solche Auffassung für unrealistisch; man kann nicht davon ausgehen, dass Menschen ihre angeborene Freiheit nur mittels eines freiwilligen und widerruflichen Versprechens aufgeben, unter der Bedingung, dass sie dafür Sicherheit und Gerechtigkeit erhalten. Vielmehr sind Gehorsam und Unterwerfung den Menschen zur Gewohnheit geworden, es fiele ihnen nicht ein, nach der Legitimität der Regierung zu fragen. Es ist nicht freiwillige Zustimmung, die eine Regierung begründet, sondern zunächst Gewalt, später Zwang, schließlich Gewohnheit. Menschen besitzen laut Hume gar nicht den Verstand, den es braucht, um eine Regierung zu wählen. Auch die Pflichten zur Loyalität gegenüber der Regierung können nicht auf Zustimmung zurückgeführt werden. Die Legitimität staatlicher Herrschaft beruht nicht auf freier Entscheidung, sondern auf ihrem Nutzen. ${ }^{3}$ Nietzsche argumentiert ähnlich, wenn auch, wie es sein Stil ist, in gröberem Ton. Im Staat sieht er eine „furchtbare Tyrannei [...] eine zerdrückende und rücksichtslose Maschinerie“, eine kriegerisch organisierte „Eroberer- und Herren-Rasse“. Die Staatsgründung auf einen Gesellschaftsvertrag zurückführen zu wollen, wäre völlig realitätsfremd. ${ }^{4}$ Die zweite Frage ist schwieriger zu beantworten. Joshua Cohen hat vor einigen Jahren eine Studie publiziert, die, so scheint es, alle Probleme der Interpretation zu lösen vermag - vorausgesetzt natürlich, man hält die Menschen generell für vernünftige Wesen guten Willens. ${ }^{5}$ Doch eben diese Voraussetzung ist das Problem, das die ganze Konzeption zum Scheitern bringen kann. Ihren Nutzen suchen zwar alle Menschen, aber nicht nach der Vorschrift der Vernunft, sondern von Begierde und Gemütsaffekten beherrscht, so lehrt Spinoza. Es kann daher keine Gesellschaft bestehen ohne Regierung, Gewalt und Gesetze, welche die Begierden der Menschen und ihren zügellosen Ungestüm mäßigen und zurückhalten. Ihre egoistische Natur können die Menschen nicht überwinden. ${ }^{6}$ Wer glaubt, Menschen könnten dazu gebracht werden, nach einer bloßen Vorschrift der Vernunft zu leben,

\footnotetext{
${ }^{2}$ Locke 1989, II, 263, § 102.

${ }^{3}$ Hume 1988, 304-324.

${ }^{4}$ Nietzsche KSA 5, 324, Genealogie der Moral, 2. Abh., Nr. 17.

${ }^{5}$ Cohen 2010.

${ }^{6}$ Spinoza 1976, Kapitel 16.
} 
der träumt vom goldenen Zeitalter der Dichter oder von einem Märchen. ${ }^{7}$ Wenn dies sich so verhält, wenn, wie es im heutigen Sprachgebrauch heißt, jeder Bürger ein potentieller Trittbrettfahrer ist, der von einem Gut profitiert, ohne dazu beizutragen, dann stellt sich wiederum die Frage, ob ein Sozialvertrag beziehungsweise der durch ihn legitimierte Staat tatsächlich gleiches Recht und gleiche Freiheit garantieren kann. Platon vertrat die Ansicht, in jedem Staat gebe es Menschen, die unheilbar und unbelehrbar böswillig seien, nicht geduldet werden könnten und zum Tode verurteilt werden müssten. ${ }^{8}$

Es mag schockieren, ähnliche Gedanken bei einem der bedeutendsten Aufklärer des 18. Jahrhunderts wieder zu finden. Denis Diderot legt in seinem Enzyklopädie-Artikel Naturrecht (Moral) ${ }^{9}$ zunächst dar, dass die scheinbar so evidente Idee eines Naturrechts alles andere als klar ist. Der Philosoph muss erst einen circulus vitiosus durchlaufen, ehe er überhaupt mit seiner Reflexion beginnen kann. Er muss sich darauf gefasst machen, sogleich mit noch schwerer lösbaren Fragen konfrontiert zu werden. Er mag das Recht definieren als Grundlage der Gerechtigkeit, doch wie soll er die Gerechtigkeit definieren? Als Pflicht, jedem das zu geben, was ihm zusteht? Doch was steht den Menschen, was einem Menschen mehr oder weniger als einem anderen zu? Wenn der Mensch im absoluten Sinn nicht frei ist, wenn äußere Umstände seine Handlungen determinieren, so sind diese doch moralisch relevant. Diderot zeichnet ein nüchternes Bild der condition humaine. Menschen haben Leidenschaften und Bedürfnisse, wollen glücklich sein, möchten gerne anderen etwas antun, was sie selbst nicht erleiden wollen; sie sind sich ihrer Bosheit durchaus bewusst. Doch was kann man Menschen vorwerfen, die von den heftigsten Leidenschaften geleitet und gequält werden, die ihr Glück nur im Unglück anderer finden, die unfreiwillig, von Natur egoistisch und böse sind, aber bereit, für ihr Tun mit dem Leben zu bezahlen? Menschen, Gute wie Böse, müssen vernünftig denken. Wer sich weigert, nach der Wahrheit zu suchen, verzichtet auf seine Eigenschaft als Mensch und muss von seinen Mitmenschen als wildes Tier behandelt werden.

Es geht Diderot also um den bösen, den asozialen Menschen; dessen Rechtfertigungsversuch - er gibt seine Existenz preis und erwirbt im Gegenzug ein Recht über die Existenz der anderen - ist inakzeptabel. Kein Mensch kann für sich allein die Frage nach dem Naturrecht beantworten. Wenn aber dem Individuum diese Kompetenz abgesprochen wird, wem steht sie dann zu? Dem Menschengeschlecht, ihm allein kann sie anvertraut werden, ist doch das Wohl aller seine einzige Leidenschaft. Jeder besondere Wille ist verdächtig, kann gut oder böse sein, nur der allgemeine Wille ist immer gut, hat nie getäuscht und wird nie täuschen. Das Individuum muss sich an den allgemeinen Willen wenden, um zu erfahren, inwiefern es Mensch, Staatsbürger, Untertan, Vater, Sohn sein soll, und wann es ihm geziemt, zu leben oder zu sterben. Der volonté générale geziemt es, die Grenzen der Pflichten festzulegen. Diderot wendet sich nun direkt an sein imaginäres Publikum: Sie haben das geheiligte Naturrecht auf alles, was Ihnen nicht von der Gattung streitig gemacht wird; sie - die

\footnotetext{
${ }^{7}$ Spinoza 1994, 9-13.

${ }^{8}$ Platon $1970 \mathrm{ff}$, Nomoi, 862e.

${ }^{9}$ Diderot OC VII, 24-29; deutsch: Diderot 1961, 378-382.
} 
Gattung - wird Sie über die Natur Ihrer Gedanken und Wünsche aufklären. Alles was Sie tun, wird groß und erhaben sein, wenn es von allgemeinem und gemeinsamem Interesse ist. Diderot spricht von einer vollkommenen Übereinstimmung, einer conformité. Doch wo lässt sich dieser allgemeine Wille konsultieren? Diderot verweist auf die geschriebenen Rechte aller zivilisierten Nationen, die gesellschaftlichen Handlungen wilder Völker, gar auf die stillschweigenden Übereinkünfte der Feinde der Menschheit. Er kommt zu folgendem Schluss: Wer nur seinem eignen Willen gehorcht, ist der Feind der Menschheit; der allgemeine Wille ist in jedem Individuum ein reiner Verstandesakt; wenn die Leidenschaften schweigen, zeigt der Verstand, was jeder von seinen Mitmenschen erwarten darf und was er ihnen schuldet; die Unterwerfung unter den Gemeinwillen ist das Band aller Gesellschaften. Der Artikel endet mit dem unmissverständlichen Urteil, diese Konsequenzen seien evident für denjenigen, der vernünftig denkt, wer aber nicht vernünftig denken wolle, verzichte darauf, Mensch zu sein, und müsse deshalb als entartetes Wesen behandelt werden.

Die einfache und offenbar doch so schwierig zu beantwortende Frage, was denn der Gemeinwille sei, wird von Diderot genauso beantwortet wie dann von Rousseau: es ist das Prinzip der Reziprozität. Wieso der Exkurs zu Diderot? Seine Argumentation ist nicht leicht nachvollziehbar. Wenn jeder Mensch von Natur ein egoistisches Wesen ist, kann das Menschengeschlecht als Ganzes davon nicht frei sein. Ebenso bleibt unklar, was es heißt, einen Menschen als entartetes Wesen zu behandeln. Der Exkurs ist nötig, weil Rousseau in der ersten Fassung des Contrat social, dem Essai sur la forme de la république, Diderots Argumente aufnimmt und kritisiert. Rousseau scheint in dieser ersten Fassung differenzierter vorzugehen als Diderot, er unterteilt die Menschen nicht mit Hilfe der Kriterien von gut und böse, vernünftig und vernunftlos. Was als des Menschen Bosheit oder Unvernunft gelten kann, rührt her von seiner Versklavung und Unterwerfung; angewiesen auf seine Mitmenschen, um seine Habgier zu befriedigen, sieht er in ihnen doch nur Konkurrenten und Feinde. Die durch gegenseitige Bedürfnisse erzeugte Kooperationsgemeinschaft ist eine trügerische Sache, von der die Menschen vergeblich ihr Glück erhoffen; sie bevorzugt die Gewinner und lässt die Verlierer im Stich. Doch die unabhängige Existenzweise, die grenzenlose Freiheit des vorstaatlichen Zeitalters sind unwiederbringlich verloren. Rousseau spricht in diesem Zusammenhang von der société générale. Diese hat, so mag es zunächst scheinen, nichts zu tun mit Diderots genre humain. Handelt es sich bei Diderot um eine höhere, unparteiische Instanz, um den vertrauenswürdigen Interpreten des Naturrechts, so bei Rousseau um die „bürgerliche Gesellschaft“, wie sie dann von Smith und Hegel beschrieben wird, um die Gesellschaft der Konkurrenz, der Wohlstandunterschiede und des steten Wandels, einer Gesellschaft, welche das Fundament ist für das universelle Wohlwollen, wie Rousseau ironisch bemerkt. Die société générale ist zwar die Schöpfung unserer gegenseitigen Bedürfnisse, aber dem Elenden, der alles verloren hat, bietet sie keine Unterstützung an, er wird zum Opfer der trügerischen Vereinigung, von der er sein Glück erhofft hat, die aber nur jene unterstützt, die bereits mehr als genug besitzen. Auf die einschmeichelnde Stimme der Natur ist kein Verlass mehr, die Unabhängigkeit, die wir von ihr erhalten haben, ist kein wünschbarer Zustand. Das glückliche Leben 
des goldenen Zeitalters war für das Menschengeschlecht stets ein unerreichbarer Zustand. Der im zweiten Discours beschriebene Zustand ursprünglicher Unabhängigkeit war, wie Rousseau jetzt urteilt, geprägt von einem wesentlichen Laster: der fehlenden Verbindung zwischen den Menschen, dem Fehlen moralischer Beziehungen.

Nun nimmt Rousseau das Wort genre humain auf, doch ist anzunehmen, dass er darunter etwas anderes versteht als sein Freund Diderot. Dieser Begriff bietet dem Geist bloß eine rein kollektive Idee an, er setzt keine wirkliche Verbindung zwischen den Individuen, die sie bilden, voraus. Man kann sogar einen Schritt weiter gehen und den genre humain als moralische Instanz, als Gefühl gemeinsamer Existenz verstehen. Man kann sich einbilden, dies Gefühl sei jenes der Humanität, das auf dem Naturrecht gründe. Beobachtet man dann jedoch, was aus der Verfassung der Menschen im Verhältnis zu ihren Mitmenschen resultiert, so zeigt sich etwas ganz anderes als das, was wir erwartet hätten. Der Fortschritt der Gesellschaft erstickt die Menschlichkeit in den Herzen und erweckt das persönliche Interesse. Das Naturrecht jedoch, das man eher als Vernunftrecht bezeichnen sollte, beginnt erst dann sich zu entwickeln, wenn die Entwicklung der Leidenschaften dessen Vorschriften ohnmächtig hat werden lassen.

Das Verdikt über Diderots Naturrechtsartikel ist deutlich genug: Der angeblich von der Natur diktierte Sozialvertrag - traitté social - erweist sich als veritable Chimäre; die Voraussetzungen sind stets entweder unbekannt oder nicht praktikabel, man muss sie notwendig ignorieren oder verletzen. Wenn die société générale, die Gemeinschaft des Menschengeschlechts, tatsächlich und nicht bloß in philosophischen Systemen existierte, würde sie sich als moralisches Wesen von den Individuen unterscheiden. Es gäbe eine gemeinsame Sprache, welche die Natur allen Menschen beibrächte und die ihre gegenseitige Kommunikation ermöglichen würde. Ebenso gäbe es ein gemeinsames Sensorium, welches der Verbindung aller Teile dienen würde. Das gemeinsame Wohl wäre mehr als das Aggregat der Einzelinteressen und die öffentliche Glückseligkeit die Quelle des privaten Glücks. Wenn folglich die menschliche Gemeinschaft in der Realität existierte, hätte Diderot recht, doch eben dies ist nicht der Fall, diese Gemeinschaft existiert bloß als philosophische Idee. Rousseau nimmt, gegen Diderot, eine „realistische“ Position ein; die Frage drängt sich auf, wie viel Realismus die Theorie des Contrat social verträgt. Die Argumente, mit denen Rousseau Diderot widerlegen will, sind überraschend, vergleicht man sie mit jenen, die er später in der definitiven Fassung des Contrat social präsentieren wird. Der unabhängige Mensch hat keinen Anlass, sich ums Gemeinwohl zu kümmern, sein Eigeninteresse weist ihm nicht den Weg zum Gesamtinteresse. Das Privatinteresse verbindet sich nicht mit dem Gemeinwohl, vielmehr schließen sich beide in der natürlichen Ordnung der Dinge gegenseitig aus. Gesetze sind für Menschen ein Joch, das sie gerne anderen auferlegen, selbst aber nicht tragen wollen. Rousseau spricht wohlgemerkt nicht vom Menschen im vorstaatlichen Zustand, dieser spielt hier keine Rolle mehr. Er übernimmt vielmehr Diderots Schilderung des Menschen, der - im staatlichen Zustand - sein Glück nur im Unglück anderer findet, der unfreiwillig egoistisch und böse ist. Dieser Mensch ist aufgeklärt und unabhängig, wie Rousseau betont; jeder 
souveräne Mensch wird so räsonieren, ebenso jede souveräne Gesellschaft, die nur sich selbst gegenüber rechenschaftspflichtig ist.

Was soll man solchen Überzeugungen entgegenhalten? Die Religion zu Hilfe ziehen zu wollen, wäre ein untaugliches Rezept. Die Menge hat für die erhabenen Begriffe des Gotts der Weisen kein Gehör, ihre Religiosität schlägt rasch um in Fanatismus und Intoleranz. Der Menschheit drohte der Untergang, würden nicht die Philosophie und die Gesetze der Wut des Fanatismus Einhalt gebieten, und könnte nicht die Stimmer der Menschen jene der Götter übertönen.

Dem Philosophen also kommt die Aufgabe zu, sich mit dem Problem auseinanderzusetzen, nicht dem Theologen, der stets nur zum Schaden der Menschheit argumentiert. Der Philosoph freilich - und hier hat Rousseau wiederum Diderot im Visier - verweist uns an den genre humain. Vermag der Gemeinwille als Ausdruck der umfassenden Vernunft und Güte des Menschengeschlechts dem Individuum zu erklären, was richtig und falsch ist? Die Rede ist immer noch vom unabhängigen Menschen, vom bindungslosen Individuum. Dieses wird zugestehen, dass es die Instanz des Gemeinwillens konsultieren kann, um zu erkennen, was Gerechtigkeit ist. Doch sie kann ihm nicht erklären, welches Interesse es daran hat, gerecht zu sein. Darum also geht es: der unabhängige Mensch ist bereit, gerecht zu sein, sobald ihm bewiesen wird, dass gerechtes Handeln in seinem Interesse liegt. Rousseau räumt ein, dass der Gemeinwille in jedem Individuum ein Akt des Verstandes ist und dass, wenn die Leidenschaften schweigen, die Vernunft zeigt, was jeder von allen anderen erwarten darf und was er ihnen schuldet. Doch, so seine rhetorische Frage: wo ist der Mensch, der sich von sich selbst separieren kann? Die Sorge um die Selbsterhaltung ist immer sein stärkstes Motiv, ja ein Gebot der Natur; wie soll man ihn zwingen, die Menschheit insgesamt in Betracht zu ziehen und sich Pflichten zu fügen, deren Bezug zur eigenen Existenz er nicht zu sehen vermag? Wo ist die Vernunft, die das Individuum anzuleiten vermöchte, sich so zu verhalten, als wäre es ein anderes?

Es bleibt immer noch zu erklären, auf welche Weise das persönliche Interesse die Unterwerfung unter den Gemeinwillen erfordert. Überdies wird aus Diderots Erörterungen nicht recht ersichtlich, welche Instanz der Mensch, der wissen will, welches Handeln richtig ist und welches falsch, konsultieren soll. Die innere Stimme? Sie ist nur ein Abbild der gesellschaftlichen Konventionen. Die Prinzipien des geschriebenen Rechts, das gesellschaftliche Handeln der Völker, die stillschweigenden Übereinkünfte der Feinde der Menschheit, von denen Diderot spricht? Unmissverständliche Antworten wird man von diesen Seiten vergeblich erwarten. Die Idee einer société générale - einer Weltgemeinschaft - bleibt stets eine Projektion. Die Menschen kennen nur ihre eigene Sozialordnung, aus dieser leiten sie dann ihre Vorstellung der société générale her. Die Errichtung kleiner Republiken gibt ihnen den Traum einer großen, weltumfassenden ein. Menschen im eigentlichen Sinn, Angehörige des Menschengeschlechts, werden sie erst, nachdem sie bereits Bürger gewesen sind. Rousseau misstraut den Kosmopoliten, jenen Menschen, die ihre Liebe zum Vaterland mit ihrer Liebe zur Menschheit rechtfertigen und sich rühmen, alle Welt zu lieben, um berechtigt zu sein, niemanden zu lieben. Begriffe der universellen Gesellschaft oder des Menschengeschlechts sind mithin ungeeignet, das 
Spezifische der politischen Gemeinschaft zu erkennen. Es gibt keine natürliche Gemeinschaft, die alle Menschen umfassen würde. Menschen - Rousseau hat dies im zweiten Discours ausgeführt - werden unglücklich und böse, indem sie gesellig werden. Die Gesetze von Gerechtigkeit und Gleichheit gelten nichts für Menschen, die gleichzeitig in der Freiheit des Naturzustands und unter den Anforderungen des Gesellschaftszustands leben. Dennoch gibt es keinen Grund zur Resignation. Aus dem Übel selbst lässt sich die Abhilfe hernehmen, die es heilt. Die Rede ist von einer neuen Form der Assoziation, geeignet für eben jene Wesen, die glauben, in vollkommener Freiheit zu leben und doch den Zwängen der Gesellschaft unterworfen sind. Die politische Gemeinschaft ist das Mittel, das, aus dem Übel der Sozialisation gewonnen, es vielleicht zu heilen vermag. Das Übel also wird zur Wunderkraft, fähig, den violent interlocuteur - Diderots unglücklich-egoistischen Menschen - eines besseren zu belehren und zu bewirken, dass aus dem wilden Banditen, der er sein wollte, die stärkste Stütze einer wohlgeordneten Gesellschaft wird. ${ }^{10}$

Rousseau beansprucht, die Probleme von Recht und Gerechtigkeit besser lösen zu können als Diderot. Dies will er mit dem Contrat social - ce petit traité, wie er bescheiden hinzufügt - beweisen. Sein Anspruch ist freilich alles andere als bescheiden. Es gilt zu belegen, dass der Vertrag für vernünftige, rational kalkulierende Menschen tatsächlich vorteilhaft ist, dass aus dem Übel der Vergesellschaftung, also der Vorherrschaft der privaten Interessen, tatsächlich die Republik, die öffentliche Angelegenheit hervorgehen kann, dass schließlich so etwas wie ein gemeinsames Interesse überhaupt existieren kann.

\section{Die separierte Gesellschaft}

Von der Notwendigkeit, zwecks Etablierung des gesellschaftlichen Friedens einen Vertrag abzuschließen, hat Rousseau bereits im zweiten Discours gesprochen. Der Mensch verliert seine Freiheit und Unabhängigkeit in dem Maße, wie neue Bedürfnisse ihn seinen Mitmenschen unterwerfen. Er wird ihr Sklave, selbst wenn er ihr Herr ist. Eifersucht, Rivalität, Konkurrenz, gegensätzliche Interessen und der Wunsch, seinen Profit auf Kosten anderer zu machen, sind Übel, die nicht nur bei der Aneignung materieller Güter, sondern auch im Kampf um soziale Wertschätzung und politische Macht einen permanenten Konflikt nähren. Vor der Erfindung des Geldes waren Boden und Vieh die einzigen Güter, die Menschen besitzen konnten. Doch Boden ist ein knappes Gut; die Überzähligen, die es nicht geschafft haben, sich einen Teil anzueignen, haben die Wahl, sich den Reichen zu unterwerfen und ihnen zu dienen, oder sie zu berauben, um überleben zu können. Die Mächtigsten beanspruchen aufgrund ihrer Stärke, die Elendesten aufgrund ihrer Bedürfnisse ein Recht auf fremdes Eigentum. Die widerrechtlichen Aneignungen der Reichen und die Räubereien der Armen führen nach einer Jahrtausende währenden Evolutions- und Zivilisationsgeschichte erst zu jenem Krieg, den Hobbes mit dem Naturzustand gleichsetzt. Die Société naissante weicht dem schrecklichsten Kriegszustand, doch der Menschheit ist die Rückkehr in einen früheren Zustand, der

\footnotetext{
${ }^{10}$ Rousseau OC III, 281-289.
} 
Verzicht auf die zivilisatorischen Errungenschaften verwehrt.

Aus diesem Kriegszustand kann nur ein Sozialvertrag den Ausweg weisen, das gesteht Rousseau seinen Vorgängern zu. Die für das Zustandekommen eines für alle Partner vorteilhaften Abkommens Wahlfreiheit, gleiche Ausgangsbedingungen, übereinstimmende Interessen - sind jedoch nicht gegeben. Die Verschärfung der Ungleichheit hat den Krieg ausgelöst; wenn der Vertrag zwecks Beendigung des Kriegs nötig ist, kann er doch angesichts ungleicher Ausgangsbedingungen nicht auf einem vernünftigen Konsens zwischen freien Menschen, sondern nur auf einem Kompromiss zwischen streitenden Parteien beruhen. Da der Krieg zwar für alle Seiten bedrohlich, aber vor allem für die Reichen kostspielig ist, drängen primär sie auf den Abschluss des Vertrags. Ihr Eigentum können sie allerdings nicht auf allgemein anerkannte Rechtstitel gründen. Ein legitimes Recht auf privates Eigentum beschränkt sich laut Rousseau auf den individuellen Lebensunterhalt, auf die unbedingt notwendigen Subsistenzmittel. Wer sich darüber hinaus vom gemeinsamen Lebensunterhalt etwas aneignen will, bedarf dazu der ausdrücklichen Zustimmung der Menschheit. Die Wohlhabenden fassen nun den genialsten Plan, der je erdacht wurde. Sie lassen die Kräfte ihrer Feinde - der Besitzlosen - für sich arbeiten und machen aus ihren Gegnern ihre Beschützer. Die Institutionalisierung einer Rechtsordnung mit staatlichem Gewaltmonopol ist als Ausweg aus einer desolaten Situation unumgänglich, perpetuiert aber eben die Ungleichheit, deren destruktive Wirkung sie beenden soll. Die Gesetze legen dem Schwachen neue Fesseln an und geben dem Reichen neue Kräfte, zerstören die natürliche Freiheit, verewigen das Gesetz des Eigentums und der Ungleichheit, verwandeln eine raffinierte Usurpation in unwiderrufliches Recht und unterwerfen um des Profits einiger Ehrgeiziger willen die Menschen der Arbeit, der Knechtschaft und dem Elend. Jede Staatsgründung ist, wie Rousseaus historische Rekonstruktion ergibt, das Werk einer privilegierten Gruppe von Menschen und dient der Absicht, die ungleiche Verteilung von Macht und Gütern auf stabilen Grund zu stellen. Staat und Gesetz schränken die Freiheit ein; dass die Armen, die nur ihre Freiheit besitzen, diese ohne Gegenleistung opfern, dass gar Völker sich bedingungslos einem absoluten Herrscher unterwerfen, ist unwahrscheinlich. Es wäre absurd, den Menschen einen angeborenen Hang zur Knechtschaft zu unterstellen. Absolute Herrschaft kann nie rechtmäßig sein. Jede Theorie, die dem Menschen zumutet, auf die Freiheit, sein vornehmstes Vermögen zu verzichten, ist Ausdruck sklavischen Sinns. Auf die Analyse des jeder Regierung zugrunde liegenden Vertrags will Rousseau in diesem Werk nicht eingehen. Er hat dabei allerdings noch ganz traditionell den Vertrag zwischen Volk und Obrigkeit vor Augen. Das Wesen des Staats besteht ihm zufolge nicht in der Obrigkeit, sondern im Gesetz. Hält sich die Regierung nicht daran, ist es für das Volk nicht bindend. Menschliche Leidenschaften werden jedoch stets stärker sein als Gesetze. Die Laster machen Institutionen erforderlich, zugleich aber deren Missbrauch unvermeidlich. Die Argumentation endet in einer Aporie: Gesetze können Menschen disziplinieren, aber nicht verändern. Eine gute, nicht korrupte Regierung wäre eine, die ebenso überflüssig ist wie Obrigkeit und Gesetz in einem Land, in dem niemand Gesetze übertritt und die Macht missbraucht. 
Die Konklusion aus dieser Geschichte ist klar. Mit den Fortschritten der Zivilisation ist der Staat zwar für das Überleben der Menschen unverzichtbar geworden, kann aber nie eine legitime Institution sein, weil er stets den Interessen der Privilegierten dient und eine Ordnung von Abhängigkeit und Ungleichheit, Konkurrenz und Korruption perpetuiert, folglich mit dem Naturrecht unvereinbar ist. Staaten werden von den Reichen und Mächtigen gegründet, und ihr einziger Zweck besteht darin, Reichtum und Macht zu bewahren sowie sicherzustellen, dass die Armen und Machtlosen diese Ordnung nicht gefährden. Rousseaus Argumentation ist von derjenigen Humes und Nietzsches gar nicht so weit entfernt. Studiert man die Zivilisationsgeschichte, ist es somit höchst unwahrscheinlich, dass man darin so etwas vorfinden wird wie einen legitimen Staat, eine gerechte Gesellschaft oder gar einen Sozialvertrag zwischen Gleichen.

Die Abhandlung mit dem Titel Du Contract social ou principes du droit politique unterscheidet sich grundlegend von Rousseaus anderen politischen Schriften. Den Abschluss eines legitimen Gesellschaftsvertrags wird man mit den Worten von Jean Starobinski nur in einer normativen Dimension außerhalb der historischen Zeit finden. ${ }^{11}$ So wäre denn der Contrat social eine Chimäre und eine Utopie? Eine einfache Antwort auf diese Frage ist nicht zu erwarten. Rousseau bemüht sich offensichtlich, sein Publikum davon zu überzeugen, eine solche Verfassung sei realisierbar. Zu bestimmen sind deren Legitimitätskriterien, wobei Rousseau zunächst einmal pragmatisch denkt: das Interesse darf nicht vom Recht, die Zweckmäßigkeit nicht von der Gerechtigkeit getrennt werden. Die Menschen sind so zu nehmen, wie sie sind; die Gesetze so einzurichten, dass sie mit der menschlichen Natur verträglich sind. Doch wie sind die Menschen, wie lässt sich die menschliche Natur definieren? Rousseau argumentiert rationalistisch: Der Mensch ist ein egoistischer Nutzenmaximierer, er wird nur Gesetze akzeptieren, die ihm nützlich sind. Diese Definition lässt die Frage offen, ob der Mensch fähig ist, sein wahres Interesse zu erkennen. Wenn wir vom zweiten Discours ausgehen, ist dies eher unwahrscheinlich. Im Konflikt zwischen dem vernünftigen amour de soi-même und dem korrumpierenden amour propre wird der zweite in der Regel den Sieg davon tragen, anders gesagt: sein wahres Interesse wird der Mensch meist verkennen. Was unterscheidet das wahre vom falschen Interesse? Ich denke, Rousseau versteht unter dem wahren Interesse den Willen zu Freiheit und Unabhängigkeit, zur individuellen Autarkie, die auch in der Gesellschaft möglich sein muss. Das falsche Interesse wäre dann der Drang nach Anerkennung und materiellen Reichtümern. Rousseau sieht den Zweck des Vertrags in der Garantie der Freiheit der Bürger, nicht in der Umverteilung der Güter. Erinnern wir kurz an die Economie Politique. Rousseau unterscheidet darin die politische von der privaten Ökonomie. In politischer Hinsicht sind die Mitglieder der ,großen Familie“ von Natur gleich. In ökonomischer Hinsicht gebührt hingegen dem Vater von Natur die Autorität. Während in der Familie alle Eigentumsrechte ihm zustehen, wird im Staat die öffentliche Verwaltung zur Sicherung des bereits bestehenden privaten Eigentums der Bürger errichtet.

\footnotetext{
${ }^{11}$ Starobinski 1971, 44-46.
} 
Menschen schließen sich zu einer politischen Gemeinschaft zusammen, um das Vermögen, das Leben und die Freiheit jedes Einzelnen durch den Schutz der Gemeinschaft zu sichern, wie Rousseau im Anschluss an Locke erklärt. Doch wie kann die Freiheit eines Individuums verteidigt werden ohne jene seiner Mitbürger zu beschränken? Wie kann für öffentliche Bedürfnisse gesorgt werden ohne auf das Eigentum der Bürger zurückzugreifen? Die scheinbar unüberwindliche Schwierigkeit kann durch das Gesetz, die erhabenste menschliche Institution behoben werden, wie Rousseau glaubt. Da in der Gesellschaft alle Verpflichtungen gegenseitig sind, ist es unmöglich, sich über das Gesetz hinwegzusetzen, ohne auf seine Vorteile zu verzichten. Rousseau erwähnt hier bereits jenes Prinzip der Reziprozität, das dann im Contrat eine zentrale Rolle spielen wird.

Eine weitere Aufgabe der Regierung besteht in der Erziehung zur Tugend. Rousseau übernimmt die republikanische Rhetorik: von den Erfolgschancen der Politik zeichnet er ein düsteres Bild. Das Überhandnehmen privater Interessen, die unsinnige Vermehrung der Gesetze, die Zunahme der Korruption und Regierungen, die nur danach trachten, das Volk zu unterwerfen und zu enteignen - all diese Gefahren drohen dem Gemeinwesen, wenn die Bürger ihre Pflicht vernachlässigen und die Tugend sich nicht behauptet. Die dringendste und schwierigste Aufgabe der Regierung besteht darin, allen Gerechtigkeit widerfahren zu lassen, insbesondere die Armen vor der Tyrannei der Reichen zu schützen. Je größer die Wohlstandsunterschiede in einer Gesellschaft sind, desto mehr nimmt allerdings die regulierende Macht der Gesetze ab. Es gehört daher zu den wichtigsten Aufgaben der Regierung, der Ungleichheit der Vermögen vorzubeugen. Dabei handelt es sich jedoch um ein höchst schwieriges Unterfangen. Die Regierung darf weder den Besitzern ihre Schätze wegnehmen noch Armenanstalten einrichten, dennoch muss sie die Akkumulation von Reichtum verhindern und die Bürger vor Verarmung bewahren. Für den Unterhalt der Bürger und die öffentlichen Bedürfnisse zu sorgen sowie die Menschen zur Arbeit anzuhalten, gehört ebenso zu den Regierungsaufgaben. Die Schwierigkeit der öffentlichen Verwaltung der Güter ergibt sich aus dem Umstand, dass das Eigentum das heiligste aller Bürgerrechte und das Fundament der bürgerlichen Gesellschaft ist. Da nur der Besitz das gesetzeskonforme Handeln der Bürger garantiert - Verstöße werden mit Geldstrafen geahndet - ist das Eigentumsrecht elementarer als die Freiheit. Staatliche Aktivitäten müssen allerdings durch die Beiträge der Bürger finanziert werden. Rousseau glaubt nicht an die Redlichkeit der Regierenden, diese verfolgen ihre privaten Interessen, sind geleitet von Habgier und Verschwendungssucht, derart untergraben sie das Vertrauen und die Steuermoral der Bürger. Erzwungene Steuern können nicht legitim sein, freiwillige sind dagegen nutzlos. Die Schwierigkeit einer gerechten und weisen Ökonomie besteht denn auch in der grausamen Alternative, entweder den Staat zugrunde gehen zu lassen oder sich am geheiligten Recht auf Eigentum zu vergreifen. Rousseau rät zu Sparsamkeit; er warnt vor der Zunahme von Bedürfnissen, die ihre Ursache in nutzlosen Begierden haben. Unter Ökonomie versteht man üblicherweise das weise Haushalten mit den vorhandenen Mitteln, nicht den Erwerb neuer Mittel, wie er zu bedenken gibt. Rousseau ruft als getreuer Schüle von Locke nochmals die fundamentale Bedeutung des Eigentums und des Rechts, es 
ungestört zu genießen, in Erinnerung. Zumindest stillschweigend hat sich mit dem Vertrag jeder Bürger verpflichtet, zur Befriedigung der öffentlichen Bedürfnisse beizutragen, doch diese Beitragspflicht ist nur legitim, wenn sie auf Freiwilligkeit beruht, auf der Zustimmung des Volks. Rousseau empfiehlt eine proportionale Kopfsteuer, die das lebensnotwendige Vermögen verschont, das überflüssige dagegen, alle Arten von Luxusgütern also, umso mehr belastet. Zu erwägen ist ihm zufolge ebenfalls der Nutzen, der den Bürgern aus der Gemeinschaft erwächst - zumindest den wohlhabenden Bürgern, wäre zu ergänzen. Die Gemeinschaft beschützt die unermesslichen Besitztümer der Reichen, während sie dem Elenden noch in seiner selbstgebauten Hütte zusetzt. Kommen nicht alle Vorteile der Gesellschaft den Mächtigen und Reichen zugute, während die Armen bloß die Lasten tragen, so Rousseaus rhetorische Frage? Von einem Gesellschaftsvertrag zwischen Gleichen kann in der Economie Politique so wenig die Rede sein wie im Discours sur l'inégalité. Der Vertrag wird zwischen zwei Ständen geschlossen oder vielmehr vom mächtigeren Stand diktiert; er lautet kurz und bündig: „Ihr seid auf mich angewiesen, denn ich bin reich und ihr seid arm. Lasst uns daher eine Vereinbarung treffen: ich werde erlauben dass ihr die Ehre habt, mir zu dienen, unter der Bedingung, dass ihr mir das wenige gebt was euch bleibt, für die Mühe die ich mir mache, euch zu befehligen. “12

Staat und Regierung sind nützliche und notwendige Einrichtungen, man könnte auch von einem notwendigen Übel sprechen; eine legitime Verfassung jedoch können sie nicht garantieren. Dieser Schluss ergibt sich aus dem Discours sur l'inégalité wie aus der Economie Politique. Das republikanische Pathos tritt im Contrat, zumindest in den ersten Kapiteln, zunächst einmal in den Hintergrund. Der Abschluss des Vertrags kommt einem vorteilhaften Tausch gleich. Dass jeder Bürger verpflichtet ist, seine Rechte bedingungslos abzugeben, betrifft die allfälligen Privilegien, die Sonderrechte, so darf beispielsweise - wie dies schon bei Locke der Fall ist - ein Recht auf Selbstjustiz nicht erlaubt werden. Rechtsgleichheit setzt gleiche Ausgangsbedingungen voraus. Sind diese gegeben, muss kein Bürger sich einem anderen unterordnen; jeder Bürger kann anderen gegenüber nur Rechte beanspruchen, die er ihnen über sich selbst einräumt. Die gleiche Abhängigkeit aller Bürger vom Gesetz soll verhindern, dass sie in private Abhängigkeit geraten. Nur der in seinem Handeln autonome Bürger ist kompetent, an der Gesetzgebung zu partizipieren. Doch dieser Bürger ist, so muss man annehmen, immer noch der rationale Egoist, der in der Vertragsgemeinschaft seinen Vorteil sucht. Spricht Rousseau von einer Veränderung, die der Ausgang aus dem Naturzustand im Menschen bewirkt, von der Vernunft, die an die Stelle der Neigung tritt, so antizipiert er Kant: Nur der Gehorsam gegenüber dem Gesetz, das man sich selber gegeben hat, ist Freiheit. Für eine rein rationalistische Konzeption ist dieser Zusatz jedoch überflüssig. Der Bürger muss ein Interesse am Bestand der Republik haben und darin seinen Vorteil erkennen. Wie bereits erwähnt, ist das Recht auf privates Eigentum für Rousseau so elementar wie jenes auf individuelle Freiheit. Was folgt daraus? Weil das Eigentumsrecht grundlegend für den Staat ist, können die Gesetze stets nur für die

\footnotetext{
${ }^{12}$ Rousseau OC III, 273.
} 
Besitzenden nützlich sein. Ideal wäre für Rousseau eine Gesellschaft, worin alle etwas, aber niemand zu viel besitzt. Der Vertrag soll die Rechtsgleichheit verwirklichen, nicht die soziale Gleichheit; unter Gerechtigkeit ist nichts anderes zu verstehen als Rechtsgleichheit. ${ }^{13}$ So unmissverständlich Rousseaus Urteil ist, ergibt sich daraus dennoch ein Problem, ist es doch gerade die soziale Ungleichheit, welche das Fundament der Republik zu unterminieren vermag. Dabei ist zunächst zu präzisieren, dass es Rousseau nicht um distributive Gerechtigkeit geht. Wenn einige Bürger weniger haben als andere, so ist diese Ungleichheit als solche für die Republik nicht gefährlich. Die Gefahr besteht vielmehr darin, dass der Vermögende den Armen korrumpieren, in seine Abhängigkeit bringen kann und der Arme aus materiellen Gründen bereit ist, sich korrumpieren zu lassen und seine Unabhängigkeit aufs Spiel zu setzen. Gefahr droht dem Staat von jenen, die im Überfluss schwimmen und vom gemeinsten Pöbel, hält doch der Reiche das Gesetz in seinem Geldbeutel, und der Arme liebt das Brot mehr als seine Freiheit, wie Rousseau in den Lettres écrites de la Montagne schreibt. ${ }^{14}$

Die ideale soziale Basis der Republik ist für Rousseau ein breiter Mittelstand - Bürger, die bezüglich Vermögen und Intelligenz ungefähr gleich sind, die keine maßlosen Ansprüche stellen, aber sehr wohl etwas zu verlieren haben. Bei solchen Menschen ist das Gesetz am sichersten aufgehoben. Dass es in jeder Gesellschaft unvermeidlich konkurrierende private Interessen gibt, war Rousseau aber natürlich klar. Die Republik kann den Konflikt dieser Interessen nur überwinden, wenn diese in einem Punkt übereinstimmen. ${ }^{15}$ Was ist das Gemeinwohl, wie lässt sich dieser so suggestive und doch so unklare Begriff definieren? Es muss sich um etwas handeln, was dem rationalen Egoisten - nicht dem moralisch „gezähmten“ Menschen - als grundlegend erscheint, worauf er unbedingt angewiesen ist. Für Rousseau ist dies ganz einfach das Prinzip der Reziprozität, der gegenseitigen Anerkennung der Bürger und ihrer Ansprüche auf Recht und Freiheit. Kommunitaristische Interpretationen sind hier völlig unangemessen; das Gemeinwohl darf keinesfalls verstanden werden als ein Gut, das wertvoller wäre als die rationalen Interessen der Individuen. Was für die Republik und die Bürger gut und richtig ist, lässt sich nur prozedural bestimmen, nicht unter Berufung auf das Naturrecht. Nur der Gesamtheit autonomer Bürger steht es zu, das Gemeinwohl zu definieren. Das heißt freilich auch für Rousseau nicht, das Volk habe immer Recht. Volksentscheide können falsch sein, dies ist vor allem dann der Fall, wenn Sonderinteressen die Urteilskraft der Bürger korrumpieren. Verpflichtungen der Gemeinschaft gegenüber sind wegen ihrer Reziprozität zwingend; wer sie erfüllt, kann nicht anderen nützen ohne zugleich sich selbst zu nützen. Wenn der Gemeinwille immer recht hat und alle stets das Glück aller wollen, so schlicht deshalb, weil jeder Bürger bei seiner Willensbekundung an sich selbst zuerst denkt. ${ }^{16}$ Jeder Bürger erkennt den Vorteil, den ihm die Rechtsgleichheit verschafft, und weiß zugleich, dass er in deren Genuss nur kommt, wenn er sie seinen Mitbürgern ebenfalls zuerkennt. Als kluger Egoist muss er kein moralisch guter Mensch sein. Wohl nirgends hat Rousseau diese

\footnotetext{
${ }^{13}$ Rousseau OC III, 891.

${ }^{14}$ Rousseau OC III, 889-890.

${ }^{15}$ Rousseau OC III, 368.

${ }^{16}$ Rousseau OC III, 373.
} 
Überzeugung deutlicher formuliert als in der eben zitierten Stelle.

Nach der Lektüre des gesamten Contrat social kann die Frage nicht ausbleiben, ob Rousseau überhaupt als Vertragstheoretiker gelten kann. Er spricht ein Problem an, das in den Vertragstheorien seiner Vorgänger ausgeblendet bleibt. Zwar teilt er die Annahme, es sei jedem Menschen möglich, dem größeren Übel des gefahrvollen Naturzustands das geringere der Unterwerfung unter die Gesetze vorzuziehen. Doch Verträge werden in fiktiven Welten geschlossen, in denen die Unabhängigkeit und rationale Kompetenz aller potentiellen Vertragspartner einfach vorausgesetzt wird. In der realen Welt sind Menschen in Abhängigkeiten gefangen, dem Druck partikularer Interessen ausgesetzt und über ihre Handlungsoptionen meist nur unzureichend informiert. Ein Gesetzgeber wird nötig, wie Rousseau knapp festhält, eine übermenschliche Intelligenz, welche die menschlichen Leidenschaften zwar kennt, aber doch frei von ihnen ist. Um einem Volk Institutionen zu geben, muss des Menschen Natur verändert werden; das autonome, sich selbst genügende Individuum muss ins soziale Ganze eingefügt werden; seine eignen Kräfte müssen ihm genommen und ersetzt werden durch solche, die er ohne die Hilfe der anderen nicht nutzen kann. ${ }^{17}$ Was motiviert den konsequenten Fürsprecher individueller Unabhängigkeit, für den der Gedanke an die Denaturierung, an die vollständige Integration des natürlichen Menschen in die soziale Gemeinschaft, geradezu masochistisch ist, zu solchen Überlegungen? Andernorts hat er festgehalten, Bürger könnten sich noch so sehr als Mitglieder des Staats wähnen, nie könnten sie sich so mit dem Staat vereinigen, wie die Glieder eines Menschen mit seinem ganzen Körper. Es sei unmöglich zu verhindern, dass jeder Bürger eine individuelle und separate Existenz besitze, kraft welcher er fähig sei, sich selbst zu erhalten. ${ }^{18}$ Rousseau argumentiert in dieser Frage widersprüchlich, wie sich dann auch im Emile zeigen wird.

Der Gesetzgeber ist eine rein fiktive Figur ist; keine übermenschliche Intelligenz wird je in die menschlichen Geschicke eingreifen. Die vertragstheoretische Hypothese, der zufolge Menschen im Moment ihres Zusammenkommens Einsicht in ihre wahren Interessen gewinnen, ist für das Verständnis der realen Geschichte der Entstehung politischer Institutionen unbrauchbar. Menschen verändern ihr Verhalten nur langsam und aufgrund langer Erfahrungen. Die Kompetenz des législateur, des Gesetzgebers, erstreckt sich weder auf die Regierung noch auf die legislative Souveränität. Die fiktive Instanz steht für die Möglichkeit, aus folgender Aporie einen Ausweg zu finden: Damit ein Volk bei der Gründung der Republik klug agiert, müsste die Wirkung zur Ursache werden. Der esprit social, der sich erst in der republikanischen Praxis entfalten kann, müsste eigentlich schon die Staatsgründung begleiten. Die Menschen müssten als Gesetzgeber über Kompetenzen verfügen, die nur aus der pädagogischen Wirkung guter Gesetze resultieren können. Der Gesetzgeber ist ein Wissenschaftler, ein Historiker und Soziologe; der philosophischen Konstruktion legitimer Herrschaft hält er die Erfahrung entgegen, dass politische Praxis von zahlreichen Faktoren sozialer, ökonomischer und kultureller Art bestimmt wird. Weichen die Bürger in ihren Interessen

\footnotetext{
${ }^{17}$ Rousseau OC III, 381-382.

${ }^{18}$ Rousseau OC III, 606.
} 
nicht zu stark voneinander ab und sind die Wohlstandsunterschiede mäßig, gibt es Chancen für eine gute Verfassung, was freilich, wie Rousseau immer wieder zu verstehen gibt, nur selten der Fall ist. Eine Gleichverteilung von Macht und materiellen Gütern ist wie bereits dargelegt nicht anzustreben, doch ist darauf zu achten, dass kein Bürger so reich ist, um sich einen anderen kaufen zu können, und keiner so arm, um sich verkaufen zu müssen. Es sind die allzu Reichen und die Bettler, die den Bestand des Gemeinwesens gefährden und die öffentliche Freiheit zu Markte tragen. Doch Rousseau will trotz all seiner Bedenken bezüglich der Chancen der Republik den Pessimisten nicht Recht geben. Die Gleichheit sei bloß eine Chimäre, Folge einer Spekulation, die in der Praxis zu nichts führe, so das übliche Vorurteil. Wenn aber der Missbrauch unvermeidlich ist, wenn der Lauf der Dinge die Gleichheit zerstört, muss die Kraft der Gesetzgebung dieser Zerstörung entgegenwirken. ${ }^{19}$ Sobald der Dienst an der Öffentlichkeit für die Bürger nicht mehr Vorrang hat, sobald ihnen die privaten Interessen wichtiger werden, steht der Staat vor dem Ruin. Ist der Mensch von Natur ein unvernünftiges Wesen, oder wird er es in der Gesellschaft? Die unvernünftigen, politisch schädlichen Verhaltensweisen des Adels hat Machiavelli in seinen Schriften immer wieder angeprangert. Rousseau beruft sich auf ihn, wenn er festhält, ein kluger Fürst müsse alles Interesse daran haben, über ein mächtiges Volk zu herrschen. Da Fürsten jedoch unklug und auf ihren persönlichen Vorteil bedacht seien, räumten sie stets jener Maxime den Vorrang ein, die unmittelbar den größten Nutzen verspricht. ${ }^{20}$ Machiavelli und Rousseau formulieren eine Theorie der Handlungsrationalität, die von jener Hobbes' abweicht. In der vertragstheoretischen Logik von Hobbes ist jeder Partner potentiell ein Trittbrettfahrer; als Nutzenmaximierer ist er versucht, vom Gesetz zu profitieren und zugleich das Prinzip der Reziprozität zu missachten, um sich zusätzliche Vorteile zu verschaffen. Wie hingegen die historische Erfahrung lehrt, sind am ehesten Menschen mit privilegiertem Status in der Lage, die Kooperationslasten auf die unteren Klassen abzuwälzen. Mit der Zunahme von Macht und Reichtum steigt die Chance, negative Folgen eigenen Handelns zu externalisieren, ebenso die Versuchung, irrational, im Widerspruch zu wohlverstandenen Eigeninteressen und den Stabilitätsvoraussetzungen politischer Gemeinschaft zu handeln. In den Lettres écrites de la Montagne hat Rousseau am Beispiel Genfs demonstriert, wie die Volkssouveränität ausgehöhlt wird. Ursprünglich sind Legislative und Exekutive nicht getrennt, später sieht das Volk sich aus praktischen Erwägungen gezwungen, Aufgaben zu delegieren. Die Regierenden neigen dazu, sich zu verselbständigen, stets mehr Handlungskompetenz zu beanspruchen, sich der Rechenschaftspflicht zu entziehen und die Kompetenzen der Legislative zu usurpieren. Auf diese Weise bricht die Exekutive den Gesellschaftsvertrag, die Demokratie geht zugrunde. ${ }^{21}$ Hinzu kommt, dass die Exekutivämter vorwiegend von Angehörigen der sozialen Oberschicht besetzt werden. Kurzum, jeder Demokratie droht eine Aristokratisierung, eine Monopolisierung der politischen Macht durch die Reichen und

\footnotetext{
${ }^{19}$ Rousseau OC III, 391-392.

${ }^{20}$ Rousseau OC III, 409; vgl. zur Unvernunft der europäischen Fürsten: 563-600, Extrait du Projet de Paix Perpétuelle.

${ }^{21}$ Rousseau OC III, 814-815; 421-423; 977.
} 
Mächtigen, die ihr eigenes Wohl zum Gemeinwohl erklären. Der Missbrauch der Macht schadet nie den Mächtigen, stets den Ohnmächtigen, wie Rousseau weiß. Angesichts der natürlichen Leidenschaften der Menschen, insbesondere der wohlhabenden Oberschicht, ist zu erwarten, dass sie ihr eigentliches, rationales Interesse, jenes an der Rechtsgleichheit, nur allzu gerne zu vergessen bereit sind, wenn sie dafür ihre - politisch gesehen: irrationalen - Interessen nach mehr Reichtum und weniger Kontrolle durch die Gesetze besser verfolgen können.

Das eigentlich politische Problem, wie das Gesetz über den Menschen gestellt werden kann, ist für Rousseau so unlösbar wie die Quadratur des Zirkels. Die Menschen mögen ihre wahren Interessen wohl kennen, ihr Handeln aber richten sie nicht nach ihnen. Was nützt es, wenn die Vernunft sie erleuchtet, aber die Leidenschaft sie leitet? ${ }^{22}$ Der Bürger von Rousseaus Republik muss kein moralisch perfekter Mensch sein, als rationaler Egoist vermag er den Vorteil von Republik, Gesetz und Rechtsgleichheit zu erkennen und zu schätzen. Doch dieses rationale Interesse steht in permanentem Konflikt mit irrationalen Interessen, mit Habgier, Ehrgeiz, Eitelkeit. Die Republik ist angewiesen auf die bürgerliche Vernunft, doch ist sie keine Erziehungsanstalt, es liegt nicht in ihrer Kompetenz, aus leidenschaftlichen vernünftige Menschen zu machen. Daher ist sie permanent bedroht: das Überhandnehmen der „,unvernünftigen“, privaten Interessen, wie sie mit jedem sozialen und ökonomischen Fortschritt notwendig einhergeht, kann ihren Untergang bedeuten.

\section{Sich von der Politik separieren.}

Wahre Politik ist ein pädagogisches Unternehmen, dies hat Rousseau von Platon gelernt; doch scheint sie genauso ein unerreichbares Ziel zu sein wie die richtige Pädagogik. Der Gouverneur muss in der Erziehung dasselbe leisten wie der Législateur in der Politik, wie dieser ist er eine fiktive Instanz. Auf die Erziehung des Menschen durch den Menschen ist wenig Verlass, ist es doch keinem Menschen möglich, die seinen Zögling umgebende Welt perfekt einzurichten. Um einen Menschen zu bilden, müsste man ein Übermensch sein, nicht ein bezahlter Lehrer, wie Rousseau im Emile zu bedenken gibt. Die Suche nach dem übermenschlichen Erzieher wäre indes so sinnlos wie jene nach dem übermenschlichen Gesetzgeber. Was bleibt als Ausweg aus dem Dilemma? Rousseaus Antwort ist die Antwort Platons: Der wahre Politiker müsste sich wesensartig von den Menschen unterscheiden; weil sich ein übermenschliches Wesen dazu nicht herablassen wird, bleibt nichts anderes, als Schriften (syggrammata) zu verfassen. ${ }^{23}$ Rousseau hat zwei verfasst, eine recht kurze über den Sozialvertrag, sowie eine ziemlich umfangreiche über die Erziehung. Emile handelt von der wichtigsten aller Künste, jener, Menschen zu bilden. Was die Intention des Buches ist - Anleitung zur idealen Erziehung oder Nachweis, dass in der Realität noch der beste Erziehungsplan scheitern muss - ist nicht ganz einfach zu bestimmen. Ich möchte hier nur auf einen Aspekt hinweisen. Der Contrat social ist ein Versuch zu zeigen, unter welchen Bedingungen die Herrschaft von Menschen über Menschen legitim sein kann.

\footnotetext{
${ }^{22}$ Rousseau CC XXXIII, 238-246, Brief an Mirabeau.

${ }^{23}$ Platon Politikos 301d-e; 268d-272d; Nomoi IV 713a-714a.
} 
Es geht darin, anders als in der Economie Politique, nicht um die Erziehung der Menschen. Wenn überhaupt eine Instanz die Menschen erziehen kann, dann ist dies nicht der législateur, vielmehr sind es die Umstände, unter denen Menschen handeln, die Sitten und Gewohnheiten, die sie annehmen. Der Emile ist dagegen eine Erziehungsschrift; zu erziehen ist nicht der politische Mensch, der Citoyen, sondern der private Mensch. Die Erziehung kann nicht einen Menschen und einen Bürger zugleich schaffen, müsste sie doch gegen die Natur wie gegen die sozialen Institutionen ankämpfen. Der Patriotismus der antiken Republiken kann nicht mehr als Vorbild dienen. Er setzte die Identifikation mit dem eignen Volk und die Gleichgültigkeit allen Fremden gegenüber voraus. Den natürlichen Menschen sieht Rousseau als ein absolutes, sich selbst genügendes Ganzes, der zivile oder politische Mensch dagegen erhält seinen Wert durch seine Beziehung zur Gemeinschaft, deren Teil er ist. Die sozialen Institutionen haben ihn denaturiert, er vermag sich nicht mehr als Individuum zu fühlen, ist in ein größeres Ganzes integriert, vollständig darin aufgegangen.

Der moderne Mensch ist jedoch kein zoon politikon mehr. Selbstverleugnung, Aufopferung für die Gemeinschaft kann von ihm nicht mehr verlangt werden. Wer in der bürgerlichen Ordnung den Vorrang der natürlichen Empfindungen bewahren will, weiß nicht was er will. Dauernd ist er im Widerspruch mit sich selbst, hin- und hergerissen zwischen Neigung und Pflicht, weder Mensch noch Bürger. ${ }^{24}$ Wie ist das zu verstehen? Kann der Mensch seine Menschlichkeit nur bewahren und entfalten, wenn er sich von der Politik fernhält? Ist der Bürger seiner Menschlichkeit vollständig entfremdet, ist ihm die Rückkehr zu ihr unwiderruflich versperrt? Rousseau will offenbar sagen, der moderne europäische Mensch lebe in einem nachpolitischen Zeitalter; es gebe keine öffentlichen Institutionen mehr, keine öffentliche Erziehung, kein Vaterland und keinen Citoyen. Er widerspricht sich aber sogleich, wenn er erklärt, die Liebe für die Angehörigen der eignen Familie, dem „kleinen Vaterland“, sei die Grundlage der Liebe zum Staat; nur der gute Sohn, der gute Gatte und der gute Vater könnten zum guten Citoyen werden ${ }^{25}$. Die Argumentation ist schwer nachzuvollziehen. Politische Erziehung kann es im 18. Jahrhundert nicht mehr geben, die häusliche Erziehung zielt auf die Festigung der patriarchalen Kleinfamilie, doch diese wiederum ist die unverzichtbare Voraussetzung des politischen Gemeinwesens. Die von Rousseau in dieser und anderen Schriften immer wieder geforderte Unterwerfung der Frau wird mit einem Zweck gerechtfertigt, den zu erreichen nicht mehr möglich ist, gibt es doch keine Vaterländer mehr und keine Bürger.

Seltsamerweise attestiert er neben dem übermenschlichen Gouverneur auch dem durchaus irdischen Vater die erforderliche pädagogische Kompetenz. Die Verantwortung, die auf ihm lastet, wiegt freilich schwer, schuldet er doch der Gattung Menschen, der Gesellschaft gesellige Menschen und dem Staat Bürger $^{26}$. Rousseau, der sich diese Verantwortung nicht aufbürden mochte, beklagt den Zerfall der Sitten, der Familie und der gesamten Sozialordnung, der, wie er überzeugt ist, leicht aufzuhalten wäre, könnten die Frauen sich entscheiden, wieder Mütter zu werden und ihren Kindern die Brust zu geben.

\footnotetext{
${ }^{24}$ Rousseau OC IV, 249-250.

${ }^{25}$ Rousseau OC IV, 699-700.

${ }^{26}$ Rousseau OC IV, 262.
} 
Der Entscheid könnte eine umfassende Reform der Gesellschaft bewirken, die Kleinfamilie erstrahlte in neuem Licht, in ihr käme die Natur wieder zu ihrem Recht. Wohlgemerkt: die Schuld trifft die Frauen; erst wenn sie wieder Mütter werden, können die Männer wieder Väter und Gatten werden ${ }^{27}$. Zeichnet sich hier eine Alternative ab zur staatlichen Politik? Sollen die Kleinfamilien zum Fundament einer neuen Sozialordnung werden? Der pädagogischen Kompetenz der Eltern traut Rousseau allerdings so wenig wie der legislativen des Volks.

Rousseaus Erziehungsschrift enthält sinnvolle Ratschläge zur vernünftigen Lebensführung. Menschen, die im Prozess der Zivilisation ihre Unabhängigkeit weitgehend verloren haben, wird erklärt, wie sie zumindest ein Stück davon sich erhalten können. Jeder Mensch ist ungeachtet seines sozialen Status in dichten Abhängigkeiten gefangen, handlungsfähig ist er nur, wenn er seine eignen Kräfte richtig einschätzt und nicht auf andere Menschen angewiesen ist. Die Freiheit ist das höchste Gut; der wahrhaft freie Mensch will nur, was er vermag und tut, was ihm gefällt. Wenn es ein Mittel gibt, die gegenseitige Abhängigkeit zwischen Menschen zu vermindern, so ist es das Gesetz ${ }^{28}$. Es geht mithin auch in der Erziehungsschrift darum, den Menschen in seiner sozialen und politischen Existenzweise ins Auge zu fassen. Dabei gilt es vor allem zur Kenntnis zu nehmen, dass alle politischen und sozialen Institutionen sich im Umbruch befinden, dass nichts mehr sicher ist. Alles ist in Bewegung, eben dieser Umstand stellt die Erzieher vor eine besondere Herausforderung. Wenn Anpassung an und Einübung in die herrschenden Konventionen für die Erziehung unerlässlich sind, warnt doch Rousseau die Erzieher davor, ihr Vertrauen in die bestehende Gesellschaftsordnung zu setzen und zu verdrängen, dass Umwälzungen unvermeidlich sind und ihre Kinder vielleicht in einer anderen Welt leben werden, in einer Welt, in welcher der Große klein, der Reiche arm und der König zum Untertan wird. Wir nähern uns dem Zustand der Krise und dem Jahrhundert der Revolutionen, wie Rousseau prophezeit. Europas Monarchien werden sich nicht mehr lange halten können. Es geht hier nicht um Hoffnungen und Wünsche nach Umsturz der Verhältnisse, es geht darum, Kinder so zu erziehen, dass sie auch in einer radikal veränderten Welt überleben und anständig leben können. Das bedeutet auch, dass Väter verantwortungslos handeln, wenn sie ihren Söhnen das Privileg des Müßiggangs in Aussicht stellen. Jeder Mensch schuldet der Gesellschaft etwas; wer, vom Schicksal begünstigt, vom Staat unterstützt wird, also auf Kosten der Gemeinschaft lebt, ist nicht besser als ein Räuber, der Passanten überfällt. Arbeiten um den Lebensunterhalt zu verdienen ist für jeden Menschen eine unerlässliche Pflicht; jeder Müßiggänger ist ein Schelm ${ }^{29}$.

Das einfache Volk bildet das Menschengeschlecht, so sieht es der Erzieher; jede soziale Rangordnung verschwindet für jene die denken, sie sehen die Leidenschaften und Gefühle, die allen Menschen gemeinsam sind. Die Reichen und Mächtigen hingegen vermögen in den Armen und Ohnmächtigen nicht ihresgleichen zu erkennen: fühlende, denkende, leidensfähige Wesen. Das Gesetz ist die erhabenste menschliche Institution, ein himmlisches Geschenk und wahres Wunder, das die Menschen

\footnotetext{
${ }^{27}$ Rousseau OC IV, 258.

${ }^{28}$ Rousseau OC IV, 307-309, 311.

${ }^{29}$ Rousseau OC IV, 468-470.
} 
in den Stand versetzt, zu gehorchen ohne beherrscht zu werden, so hat Rousseau in der Economie Politique geschrieben. Ganz anders tönt es im Emile: Im Gesellschaftszustand gibt es bloß eine chimärische, nutzlose Rechtsgleichheit. Die Mittel die dazu bestimmt sind sie zu erhalten, dienen dazu sie zu zerstören. Der Mächtige missbraucht die öffentliche Macht, um den Machtlosen zu unterdrücken und das natürliche Gleichgewicht zwischen den Menschen zu stören. In allen Ländern besteht der allgemeine Geist der Gesetze darin, den Starken gegenüber dem Schwachen, den Wohlhabenden gegenüber dem Habenichts zu begünstigen. Dieser Nachteil ist unvermeidlich, und es gibt davon keine Ausnahme. Stets wird die große Mehrheit einer kleinen Minderheit geopfert und das öffentliche Interesse dem privaten unterliegen. Die täuschenden Begriffe der Gerechtigkeit und der Unterordnung dienen bloß dazu, mit Gewalt die ungerechten Verhältnisse aufrecht zu erhalten. Die Rangordnung, die angeblich allen nützt, nützt nur den Privilegierten selbst ${ }^{30}$.

Emile und Contrat social sind ungefähr gleichzeitig publiziert worden, doch die Lektüre des Emile ruft zuweilen den Eindruck hervor, angesichts der tatsächlichen Bedingungen der Ständegesellschaft seien die Ideen des Contrat bloß Illusionen und leere Versprechen. Wird im Contrat die Möglichkeit einer legitimen Republik behauptet, so verblasst im Emile dieser Traum. Der Gouverneur schickt Emile auf Reisen, um die politischen Institutionen anderer Länder kennen zu lernen. Das Buch schließt mit Emiles Erfahrungsbericht ${ }^{31}$. Gelernt hat er, der von seinem Lehrmeister zur Freiheit erzogen worden ist, dass dies wertvolle Gut stets gefährdet ist. Die Menschen streben nach Unabhängigkeit und werden dabei zu Sklaven. Emile hat die Ketten der Meinung gesprengt, jene der Notwendigkeit, der Natur, der Bedürfnisse und Leidenschaften, damit auch der Abhängigkeit von anderen Menschen, vermag er nicht zu sprengen, sie beschränken ihn nicht in seiner Freiheit. Der Gouverneur, zufrieden mit seinem Erziehungswerk, ruft Emile in Erinnerung, es sei vergeblich, von den Gesetzen die Bewahrung der Freiheit zu erhoffen; die Institutionen sind stets Instrumente der stärksten Privatinteressen. Nur das Gesetz des Gewissens und der Vernunft vermag ihn in seiner Suche nach Freiheit zu leiten, einer Freiheit, die sich in keiner Regierungsform sondern allein im Herzen des freien Menschen findet.

Über hunderte von Seiten hinweg hat Rousseau beschrieben, wie ein imaginärer Erzieher einem realen Menschen die Fähigkeiten beibringt, die es braucht um in einer schlechten Welt der Unfreiheit und des falschen Scheins ein anständiges Leben zu führen. Gibt es ein richtiges Leben im falschen? Die Frage wird auch im unvollendeten Briefroman Emile et Sophie ou les solitaires ${ }^{32}$ nicht schlüssig beantwortet. Wenn das umsichtige Erziehungswerk seine Vollendung im vollkommenen Glück von Emile und Sophie findet, erweist sich dieses schon bei den ersten Kontakten mit der realen Welt als ephemer. Sophie verliert ihre Tochter; Emile hat in Paris Geschäfte zu besorgen und nimmt Sophie mit in diesen Abgrund von Laster und Vorurteilen. Beide geben sich den Vergnügungen und Versuchungen der Großstadt hin und leben sich auseinander. Sophie erliegt dem Charme eines anderen, wird von ihm

\footnotetext{
${ }^{30}$ Rousseau OC III, 248; vgl. 310; IV, 524f.

${ }^{31}$ Rousseau OC IV, 855-860.

${ }^{32}$ Rousseau OC IV, 881-924.
} 
schwanger. Emile verlässt Paris, findet wieder zu sich und fühlt angesichts der desolaten Lage die Kraft der Erziehung, die er genossen hat; er fügt sich den Gesetzen der Notwendigkeit. Auf sich allein gestellt wird er zum neuen Menschen; mit stoischem Gleichmut nimmt er sein Geschick auf sich, ohne Bindung, ohne Familie, ohne Heimat zieht er hinaus in die Welt. Der Citoyen wird zum Menschen, der sich der ganzen Erde verbunden fühlt, zum Wandergesellen, der überall Arbeit findet, weil er sich für jede eignet, zum Vagabunden, der überall und nirgends zuhause ist, schließlich zum Matrosen. Das Schiff auf dem er angeheuert hat wird von Korsaren gekapert, die Passagiere in Alger auf dem Sklavenmarkt verkauft. Emile, zur Freiheit erzogen, gerät in Sklaverei im Wortsinn. Seine stoische Weisheit lässt sich nicht beirren: sind wir nicht stets Sklaven der Notwendigkeit, und kann die Unterwerfung unter Menschen schlimmer sein als jene unter die Natur? Das brutale Verhalten eines Aufsehers lässt er sich nicht gefallen und organisiert einen Aufstand. Dieser misslingt, doch gelingt es Emile, den Herrn davon zu überzeugen, dass nur die maßvolle Ausbeutung der Arbeitskraft ökonomisch profitabel ist. Aufgestiegen zum Sklavenaufseher, wird er berühmt in ganz Alger und endlich als Sklave des Deys dessen Regierungsberater - ein Multitalent sozusagen, der sich auf alles versteht, nur nicht darauf, seine Freiheit zu verteidigen.

Der Contrat social scheint stellenweise eher der Vergangenheit zugewandt zu sein. Dagegen weist der Emile in eine, wenn auch imaginierte, Zukunft. Diese ist aber doch nichts anderes als die Fortsetzung jener Jahrtausende währenden Geschichte der Entfernung und Entfremdung des Menschen von der Natur, seiner Domestizierung, seiner Umwandlung in ein Wesen, das fähig und bereit ist für den Verkehr mit seinesgleichen. Jeder Mensch bedarf des Anderen, jeder Mensch sucht sich den Anderen anzueignen. ${ }^{33}$ Es sind die politischen und sozialen Institutionen, die diese Domestizierung erzwingen. Doch seltsamerweise scheint Rousseau gleichzeitig anzunehmen, dass diese Institutionen ihre integrierende Kraft im 18. Jahrhundert zunehmend verlieren und eine „postpolitische“ Periode anbricht, worin die Kleinfamilie die wichtigste soziale Institution ist. Kündigt sich gar eine Rückkehr zur Natur, zur Familie als ,natürlicher“ Ordnung an?

Der Einfluss von Rousseau auf die heutige politische und Sozialphilosophie ist beträchtlich. Céline Spectors Au prisme de Rousseau gibt einen breiten Überblick über die zeitgenössischen Debatten. So unbestreitbar jedoch die Wirkung von Rousseau ist, so haben doch seine modernen Schüler - Rawls, Habermas, Cohen, Taylor, Shklar, Barber, Balibar, um nur diese zu nennen - mit ihren konstruktiven und normativen Bemühungen Rousseaus Botschaft in gewisser Hinsicht verraten. Der Sozialvertrag wird interpretiert, diskutiert, expliziert, es wird nach möglichen Anwendungen gesucht. Verdrängt wird dabei in der Regel aber, dass Rousseaus eigene Ansicht zur Möglichkeit eines solchen Vertrags alles andere als optimistisch ist.

\footnotetext{
${ }^{33}$ Rousseau OC IV, 56-57, Manuscrit Favre.
} 
Literatur:

Bloch 2010, Michael Bloch, Möglichkeit und Unmöglichkeit internationaler Politik. Rousseaus

Auffassung des Krieges, in: Deutsche Zeitschrift für Philosophie 58, 2010, 288-306.

Cohen 2100, Joshua Cohen, Rousseau, A Free Community of Equals, Oxford.

Diderot OC, Denis Diderot, CEuvres complètes, édition critique et annotée par J. Fabre, H. Dieckmann, Jacques Proust, Jean Varloot, Paris, 1975 ff.

Diderot 1961, Denis Diderot, Philosophische Schriften, Erster Band, Berlin.

Hume 1988, David Hume, Politische und ökonomische Essays, Teilband 2, Berlin.

Locke 1989, John Locke, Zwei Abhandlungen über die Regierung, Frankfurt/M.

Nietzsche KSA, Friedrich Nietzsche, Sämtliche Werke, Kritische Studienausgabe, Berlin, München

1980.

Platon Werke, griechisch und deutsch, Darmstadt $1970 \mathrm{ff}$.

Rousseau OC, Jean-Jacques, Euvres Complètes, Paris 1959 ff.

Spector 2011, Céline Spector, Au Prisme de Rousseau: usages politiques contemporaines, Oxford.

Spinoza 1976, Baruch de Spinoza, Theologisch-Politischer Traktat, Hamburg

Spinoza 1994, Baruch de Spinoza, Politischer Traktat, Hamburg.

Starobinski 1971, Jean Starobinski, Jean-Jacques Rousseau, La transparence et l'obstacle, Paris. 
